Cite this: DOI: $00.0000 / x x x x x x x x x x$

Accepted Date

DOI: $00.0000 / x x x x x x x x x x$

\title{
Dynamics of Fiberboids
}

\author{
Antony Bazir, ${ }^{a}$ Arthur Baumann, ${ }^{b}$ Falko Ziebert, ${ }^{c}$ and Igor M. Kulić ${ }^{b, d}$
}

\begin{abstract}
Fiberboids are active filaments trapped at the interface of two phases, able of harnessing energy (and matter) fluxes across the interface in order to produce a rolling-like self-propulsion. We discuss several table-top examples and develop the physical framework for understanding their complex dynamics. In spite of some specific features in the examples studied we conclude that the phenomenon of fiberboids is highly generic and robust across different materials, types of fluxes and timescales. Fiberboid motility should play a role from the macroscopic realm down to the micro scale and, as recently hypothesized, possibly as a means of biological self-propulsion that has escaped previous attention.
\end{abstract}

\section{Introduction}

Self-propulsion, the motion of objects using internal or external energy sources or fluxes, is a hallmark of living objects and an active subject of nonequilibrium physics and soft matter research. While bacterial motion 1 inspired artificial microswimmers have been abundantly proposed and investigated ${ }^{2}$, examples for manmade substrate-based self-propellers are still rather scarce. Examples on the micro-scale comprise Quincke ${ }^{3}$ and magnetic (fluid interface) rollers ${ }^{4}$ and on the macro-scale shaken granular rods ${ }^{5}$, Marangoni-driven droplets ${ }^{6}$ or Leidenfrost wheels ${ }^{7}$. We here would like to add a whole class of more examples to the latter, using a very simple and generic strategy to motorize fibers confined at non-equilibrium interfaces ${ }^{8}$.

When two bulk phases meet at an interface, more often than not, they are in a mutual thermodynamic disequilibrium. As a consequence, they exchange fluxes of energy and matter along the normal to the interface. If now a deformable filament is trapped in the interface plane, and if it is elastically responsive to the agent flowing across it, something truly astonishing happens: the filament bends into an arc, starts to axially "spin" and move along the interface, transforming itself from a passive object into a simple engine, which we here term "fiberboid". Some extremely simple, table-top experimental realizations of fiberboids are shown in Fig. 1 .

The bidirectional active motion of fiberboids (albeit with a focus on the unidirectional rotational motion of such fibers closed

\footnotetext{
a Institut Lumière Matière, UMR 5306, Université Lyon 1-CNRS, 69622 Villeurbanne, France.

${ }^{b}$ Institut Charles Sadron, CNRS, 23 rue du Loess, BP 84047, 67034 Strasbourg, France. ${ }^{c}$ Institute for Theoretical Physics, Heidelberg University, Philosophenweg 19, 69120 Heidelberg, Germany; E-mail: ziebert@thphys.uni-heidelberg.de

${ }^{d}$ Leibniz Institute for Polymer Research (IPF), 01069 Dresden, Germany; E-mail: kulic@unistra.fr
}

into a torus) was described in a recent work ${ }^{8}$. Yet our understanding of their general physics, in particular their dynamics, remains incomplete. Due to their self-organized nature that involves symmetry breaking, self-propulsion, bi-directionality of motion, interactions and collective effects, fiberboids are significantly more complex than their unidirectional variants closed into a torus or spirals ${ }^{8}$. Yet, at the same time, fiberboids are extremely simple to generate as demonstrated by the examples shown in Fig. 1 and detailed in the next section.

In this work we will distill the generic features of fiberboid motility and contrast them to more particular features exhibited by specific experimental realizations. The outline of this work is as follows. After discussing two distinct examples in section 2. in section 3 we develop a simple dynamical model describing fiberboid motion. Comparing the predictions of this toy model to experimental data, we will see how specific observations for different fiberboid types naturally lead to model extensions. In section 4 we study the "social" behavior of fiberboids, i.e. how they interact with obstacles and other fiberboids, paving the way to studying their collective behavior in the near future.

\section{Examples}

To get a taste for the fiberboid phenomenon, let us start with two easy to reproduce table-top examples. Fig. 11) shows snapshots of a nylon fiber - a piece of commercial fishing line - on an aluminum plate heated at $\sim 140^{\circ} \mathrm{C}$. Fig. 13) displays a so-called kymograph (space-time plot generated from time-lapse images as shown in part a), clearly evidencing a rolling motion along the substrate with a typical velocity of few $\mathrm{cm} / \mathrm{s}$. The direction of motion with respect to the fiber curvature depends on the coupling of the thermal gradient through the fiber (between the heated plate and the colder surroundings) to the fibers elastic deformation, cf. Fig. 2, i.e. it is determined by the sign of the coefficient of thermal expansion. For thermally contracting materials as ny- 
a

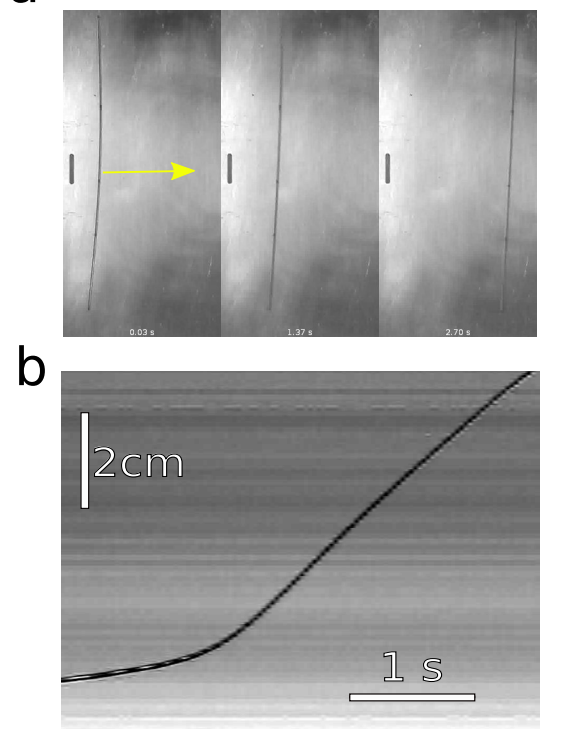

C

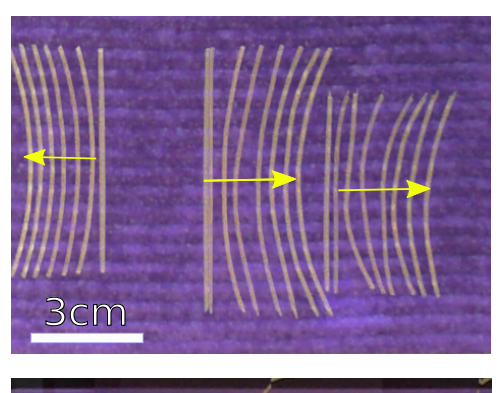

d

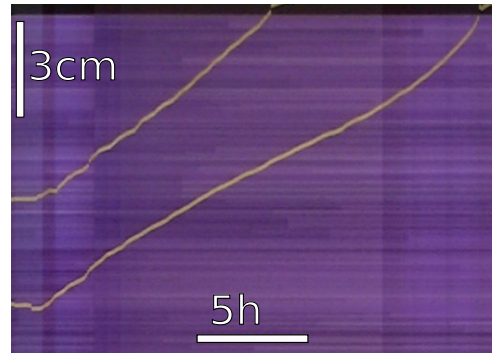

e

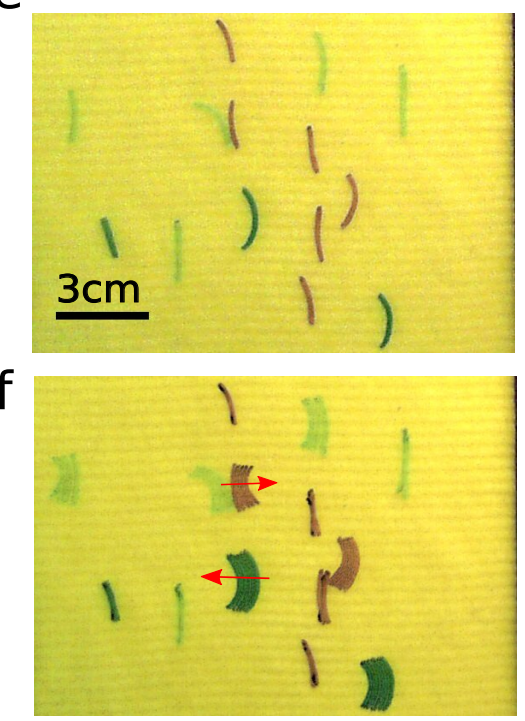

Fig. 1 Fiberboids in action. a) A nylon fiber rolling on a heated aluminum substrate. See supplementary movie 1. b) A kymograph (space vs. time plot) of the center of mass position of the nylon fiber shown in a). c) A stroboscopic picture of three initially dry spaghetti curving and then rolling on a wet towel (at $\sim 60 \%$ air humidity at $25^{\circ} \mathrm{C}$ ). See supplementary movie 2 . d) Kymographs for two of the rolling spaghetti shown in c). e) Polyacrylamide (PAM) hydrogel rods on a wet towel. f) Stroboscopic picture showing the rolling motion in e).

lon, the fiber curves away from the direction of motion, while for thermally expanding materials, such as polydimethylsiloxane (PDMS) fibers, it curves into the direction of motion ${ }^{8}$. The same effect has been recently applied to propel mono-domain liquid crystalline elastomer rods 9 .

Figs. 11)-f) show a second possibility to drive fibers: here, uncooked spaghetti (c), as well as Polyacrylamide (PAM) hydrogel rods (e), were put on a wet kitchen towel. The water then slowly enters the rods via the wet substrate, differentially swells and hence curves them. As in the case of heat flow in the first example, there is a constant solvent flow (water enters from the bottom and evaporates at the top) and the fibers start to roll, albeit much slower here, with typical velocities of few $\mathrm{cm} /$ hour, cf. the kymograph in Fig. 1 1 d). The traveling direction with respect to curvature for hygroscopic swelling is analogous to the thermal expansion case. A related effect was reported earlier using PDMS rods $\frac{10111}{1}$. However, there, a solvent droplet was injected at what later becomes the back of the rolling rod, i.e. the spontaneous symmetry breaking and bidirectionality, characteristic for fiberboids (cf. Ref. ${ }^{8}$ and as discussed below), was absent in these earlier examples.

\section{Generic models}

Looking at these examples, with different driving mechanisms and operating at (vastly) different timescales, a natural question arises: Is there a unified approach to understand the dynamics? Both systems have in common that there is a propelling agent "flowing" between two phases (baths) through the filament. The propellant, which stands for heat energy density or water concentration, respectively, in the two example cases, has a scalar density within the material denoted by $\psi(\rho, \phi, t)$. The latter depends on the polar coordinate position $(\rho, \phi)$ in the fiber cross-section in the laboratory frame and on time $t$. For the basic physics, however, only the differences of $\psi$ in the normal (z-direction/top-tobottom) and in-plane direction (x-direction/left-to-right) will be important, cf. Fig. 2 .

The responsiveness of the filament is related to the coupling between the propellant density and elastic deformations. In the simplest case, the presence of the propellant gives rise to an isotropic material eigenstrain (also called prestrain) tensor

$$
\varepsilon_{i j}^{e i g}=\alpha \psi \delta_{i j},
$$

with $i, j \in x, y, z$ and $\delta_{i j}$ the Kronecker delta. Here $\alpha$ is an isotropic linear expansion coefficient, describing thermal expansion or gel swelling, respectively. Note that this coefficient can also be negative, corresponding to contraction, as in case of heated nylon. The eigenstrain can be seen ${ }^{12 \mid 13}$ as the locally preferred strain in the absence of local and global constraints. It evolves in time with the propellant concentration and gives rise to all mechanical effects in fiberboids.

Overall the propellant's evolution is governed by three processes: (i) the propellant influx rate $q$, (ii) its relaxation rate $r$ and (iii) the rotation of the cross-section with angular velocity $\omega$, in case the rolling motion has set in. In the absence of drive, the propellant evolves via a diffusive process, heat diffusion and swelling agent diffusion in the two examples. By scaling, the associated time scale $\tau$ is determined by the diffusion coefficient of the propellant, $D_{\psi}$, and the dimension (i.e. radius) of the fiber cross-section, $R$, defining the propellant's diffusive relaxation rate

$$
r=\frac{1}{\tau} \sim D_{\psi} R^{-2} .
$$



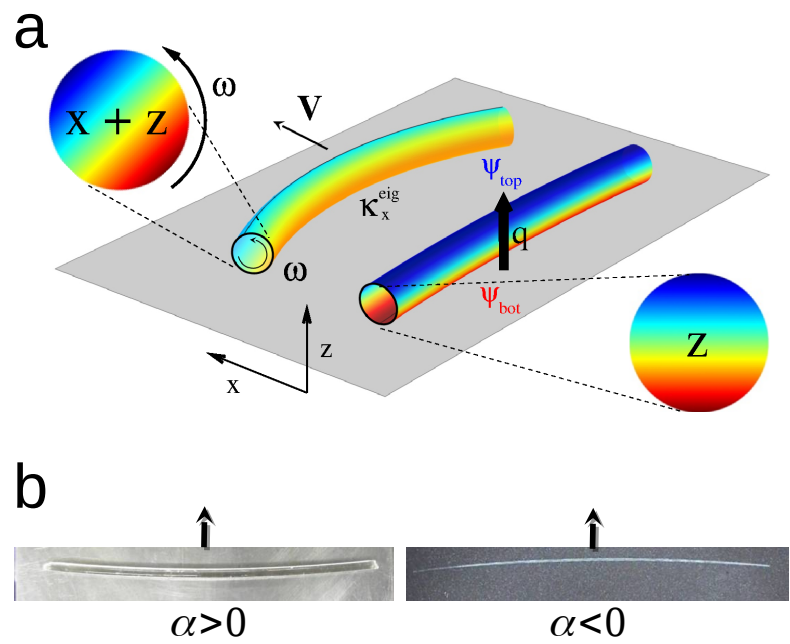

Fig. 2 a) Sketch of the mechanism underlying fiberboid rolling. The difference in propellant density $\psi_{\text {top }}-\psi_{\text {bottom }}$ gives rise to a propellant flux establishing a gradient along the z-direction. Differential expansion then leads to a preferred out-of-plane eigen-curvature, which however cannot be realized due to the confinement to the interface. Instead, an in-plane curvature $\kappa_{x}^{e i g}$ is built-up and the constant phase difference between the propellant-induced strain and the realized strain results in a driving torque that induces a rotation of every cross-section with frequency $\omega$. b) Depending on their sign of the expansion coefficient $\alpha$, fiberboids fall into two classes: "vexers" having $\alpha>0$ and rolling towards their center of curvature, as exemplified by a PDMS rod. And "cavers" having $\alpha<0$ and moving away from it, as exemplified for a nylon fiber. Arrows show direction of motion.

In the driven case, the influx rate then scales as

$$
q \sim D_{\psi} R^{-2} \Delta_{\uparrow} \psi
$$

i.e. it is given by the same characteristic time scale but in addition is proportional to the difference of the propellant concentration $\Delta_{\uparrow} \psi=\psi_{\text {top }}-\psi_{\text {bottom }}$ (between the top and the bottom of the fiber). In the absence of rotation, the propellant diffusive relaxation rate $r$ counterbalances this influx, giving rise to a typical z-gradient $\nabla_{z} \psi \sim \frac{q}{R r}$. The latter induces an eigenstrain gradient in the axial component (i.e. $\varepsilon_{y y}^{e i g}$, where $y$ lies along the fiber axis), which in turn leads to a preferred out-of-plane eigen-curvature,

$$
\kappa_{z}^{e i g} \sim R^{-1} \Delta_{\uparrow} \varepsilon_{y y}^{e i g} .
$$

This curvature $\kappa_{z}^{e i g}$, however, is entirely "virtual", i.e. it cannot be actually realized due to the presence of normal forces ${ }^{m}$ confining the fiber to the interface plane - in the two macroscopic examples presented in Fig. 1, the fiber is confined simply by its

* Two generic cases of confinement may occur: In the case of short range, adhesive contact forces - like e.g. Pickering-type pinning at the interface ${ }^{14}$ - the fiber stays fully confined as long as its bending energy density is smaller than the gain in adhesion energy. For macroscopic fibers confinement is due to gravity, which is a small and long-ranged force and additional effects come into play that can be estimated as follows: a fiber of length $l$ that is confined to the plane reduces its potential energy $e_{\text {pot }} \sim \rho_{0} g l^{3} R^{2} \kappa_{z}^{\text {eig }}$, with $\rho_{0}$ the density and $g$ the gravitational acceleration, but increases its bending energy by $e_{\text {bend }} \sim l Y R^{4}\left(\kappa_{z}^{e i g}\right)^{2}$. Matching the two energies yields own weight. Given the confinement, a fiber that is constantly driven by a flux normal to the confinement plane is mechanically frustrated 15 . Being unable to simply leave the plane, it follows an alternative path of least action and tends to axially rotate by $\sim 90^{\circ}$, so that it can realign its preferred curvature with the $\mathrm{x}$ direction.

This results in the build-up of an in-plane curvature

$$
\kappa_{x}^{e i g} \sim R^{-1} \Delta_{\leftrightarrow} \varepsilon_{y y}^{e i g},
$$

where $\Delta_{\leftrightarrow}$ now stands for the left-right difference (in-plane, perpendicular to the fiber axis). Since the eigenstrain is proportional to the linear expansion coefficient $\alpha$, fiberboids can be divided into two classes: "vexers" having $\alpha>0$ and "cavers" having $\alpha<0$ (implying convex and concave shapes, respectively) as shown in the snapshots in Fig. 2p).

The frustration-induced in-plane curvature is the precursor for the occurrence of the torque driving fiberboid rolling: the constant phase difference between the propellant-induced strain (normal to the plane) and the realized strain (in-plane) results in a driving torque that induces a rotation of every cross-section with frequency $\omega$. The driving torque density (per unit length) can be calculated from slender rod theory, as demonstrated in appendix A. By scaling, it is given as the product of the geometrically induced strain $\Delta_{\leftrightarrow} \varepsilon_{y y}^{e i g}$ and the thermally induced stress $Y \Delta_{\downarrow} \varepsilon_{y y}^{e i g}$, with $Y$ Young's modulus, integrated over the cross-section (i.e. times $R^{2}$ ), yielding

$$
m_{\text {drive }} \sim R^{2} Y\left(\Delta_{\leftrightarrow} \varepsilon_{y y}^{e i g}\right)\left(\Delta_{\downarrow} \varepsilon_{y y}^{e i g}\right) .
$$

Since the eigenstrain and the expansion coefficient $\alpha$ are linearly related and $m_{\text {drive }}$ is quadratic in strain, the torque is insensitive to the sign of $\alpha$. Together with the sign sensitivity of the in-plane curvature $\kappa_{x}^{\text {eig }}$, this explains the opposite relations of fiber curvature and direction of motion for vexers and cavers.

\subsection{Minimal model}

A minimal dynamic model, capturing the physical essence of most fiberboid phenomena has three variables: the two nondimensional eigenstrain gradients $x=R \kappa_{x}^{\text {eig }}, z=R \kappa_{z}^{\text {eig }}$ and the an-

a characteristic confinement length

$$
l_{\text {conf }}=R\left(\frac{Y \kappa_{z}^{e i g}}{\rho_{0} g}\right)^{1 / 2} .
$$

Hence, unlike for the contact force case, where the whole fiber is either fully confined or not, in the gravity case both ends of the fiber are always unconfined on a typical length scale $l_{\text {conf }}$. For long fibers with $l \gg l_{\text {conf }}$ these boundary effects will be negligible. For typical values $\left(Y=100 \mathrm{MPa}, g=10 \mathrm{~m} / \mathrm{s}^{2}, \rho_{0}=1.5 \mathrm{~g} / \mathrm{cm}^{3}, R=0.3 \mathrm{~mm}\right.$, $\kappa_{z}^{\text {eig }}=1 \mathrm{~m}^{-1}$ ) the size of the unconfined fiber ends $l_{\text {conf }} \approx 2.5 \mathrm{~cm}$ is shorter than the fiber lengths of $l=10-20 \mathrm{~cm}$ typically used in our experiments, yet not entirely negligible. This is also the probable reason for filaments shorter than few $\mathrm{cm}$ being unable to move and for sometimes occurring oscillatory behavior of the velocities of longer filaments. For simplicity, in the following we will ignore these effects assuming the fibers to be perfectly confined. 


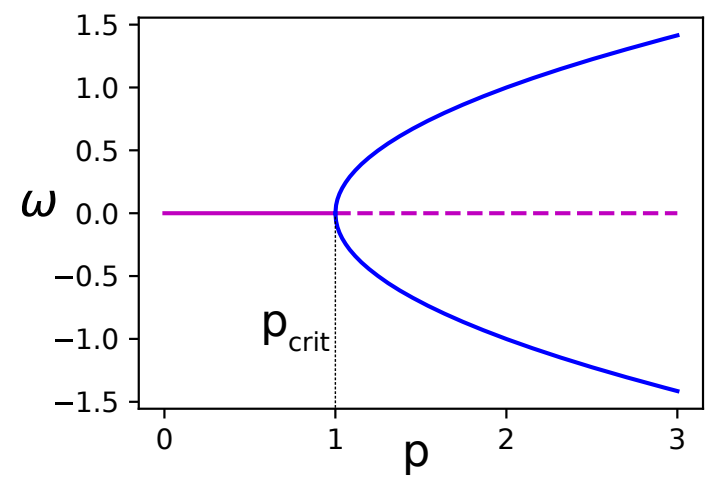

Fig. 3 The steady state angular velocity $\omega$ of a fiberboid cross-section as a function of the driving rate $p=\alpha q$, displaying a typical supercritical pitchfork bifurcation. Parameters: $r, \mu=1$. The trivial state $\omega=0$ looses stability at a critical driving strength $p_{\text {crit }}$ and the direction of rolling (reflected by the sign of $\omega$ ) is chosen by spontaneous symmetry breaking.

gular frequency $\omega$ :

$$
\begin{aligned}
\dot{x} & =-r x-\omega z \\
\dot{z} & =-r z+\omega x+p \\
\omega & =-\mu x z
\end{aligned}
$$

with constants $[r],[p],[\mu]=s^{-1}$ having dimension of a rate. The first two equations describe the kinetics of the propellant-induced eigenstrains in the cross-section, with $r$ and $p=\alpha q$ the strain relaxation and strain pumping rates, respectively. The terms proportional to $\omega$ in Eqs. (78) originate from the inter-conversion of the two modes via rotation. Eq. (9) reflects the torque balance between a, here assumed Stokes-like, dissipational torque $\propto-\xi \omega$, with $\xi$ a friction constant, and the driving torque (density) from Eq. (6), which reads $R^{2} Y x z$. The ratio

$$
\mu \sim \frac{Y R^{2}}{\xi}
$$

occurring in Eq. (9) can be interpreted as a "mechanical mobility" that captures the elastodynamic response rate of the fiber. We note that equations Eqs. (7/8), as well as the functional dependence of the driving torque can also be derived directly from the underlying equations of thermo-elasticity of a slender rod and thermal advection-diffusion ${ }^{8}$.

Let us study the steady states of the model. First, obviously, an immobile state $\omega=0$ exists, with $x=0$ and $z=p / r$. In the case of thermal driving this state corresponds to the thermal conduction state in absence of any advection. A second steady state emerges, provided the following relations hold

$$
\begin{aligned}
\omega^{2} & =r^{2}\left(A^{1 / 2}-1\right) \\
x^{2} & =\frac{r}{\mu}\left(A^{1 / 2}-1\right)
\end{aligned}
$$

where $A$ is the (dimensionless) activity parameter given by

$$
A=\frac{\mu p^{2}}{r^{3}}
$$

For the motile state to exist, the r.h.s. of Eqs. (11) and (12) have to be positive. This requires that the activity has to be larger than unity, $A>1$, implying a supercritical pumping rate

$$
|p|>p_{\text {crit }}=\mu^{-1 / 2} r^{3 / 2} \text {. }
$$

Physically, the latter relation states that the pumping rate has to be larger than a threshold value, namely large compared to the two forms of losses: $r$ (thermodynamic loss due to propellant relaxation) and $\mu^{-1}$ (frictional loss). The other way round, motion will stop, if the mobility $\mu$ drops below a critical value $\mu_{\text {crit }}=r^{3} p^{-2}$, e.g. in case when the friction increases due to a change of environment. Note that while the translational velocity $v=R \omega$ always grows with $p$ and $\mu$, the in-plane curvature $\kappa=\frac{x}{R}$ is non-monotonous in $\mu$ and vanishing both for small $\mu \leq \mu_{\text {crit }}$ and large mobilities $\mu \rightarrow \infty$. A maximum curvature $\kappa^{*}=\frac{|p|}{2 r} R^{-1}$ is attained at an intermediate mobility value $\mu^{*}=4 \mu_{\text {crit }}$.

The model hence predicts a pitchfork bifurcation in the (angular) velocity at $p=p_{\text {crit }}$, cf. Fig. 3. The rolling direction is spontaneously chosen, as observed for both the thermal ${ }^{8}$ and the hygroscopic fiberboids. Fiberboids are hence bidirectional motors and the onset of motion occurs via a spontaneous symmetry breaking. It should be noted that in Ref. ${ }^{[}$, dry friction instead of Stokes friction was used in the model, which renders the onset of motion discontinuous.

To experimentally probe the model predictions, Eqs. (11) and 12. can be used to obtain the following steady state relation

$$
|\kappa|=\frac{p|\omega| / R}{\omega^{2}+r^{2}}
$$

connecting the two directly measurable variables, curvature $\kappa$ and angular velocity $\omega$. It should be noted that the validity of this relation is independent of $\mu$ and hence of the frictional mechanism - i.e. the same relation can be obtained if dry friction is assumed in Eq. 9

Fig. 4 shows experimental results for both thermally driven nylon fibers in (a) and humidity-driven thin spaghetti (capellini) in (b). On the one hand, all the measured points, obtained both from transient dynamics and steady rolling, fall on the master curve given by Eq. 15 . This hence allows to extract the pumping and relaxation rates $p$ and $r$, as given in the figure caption.

On the other hand, an interesting question arises: why do the points, even when obtained for a single sample, not condense into a single spot? In fact, a given mechanical mobility $\mu$ should select a single point on the master curve. This can be evidenced as in Fig. 5, where numerically obtained trajectories from Eqs. (719) are shown in the plane in-plane eigenstrain vs. angular velocity. All trajectories were initiated with zero strain (and hence curvature) and velocity. After a transient excursion they all land on the

$\dagger$ Divide Eq. 12 by Eq. 11 to obtain $|x|=\frac{|\omega|}{\sqrt{\mu r}}$, then use Eq. 11. to get $\omega^{2}+r^{2}=$
$r^{2} A^{1 / 2}=\sqrt{\mu r}$, insert and use $|x|=R|\kappa|$. 

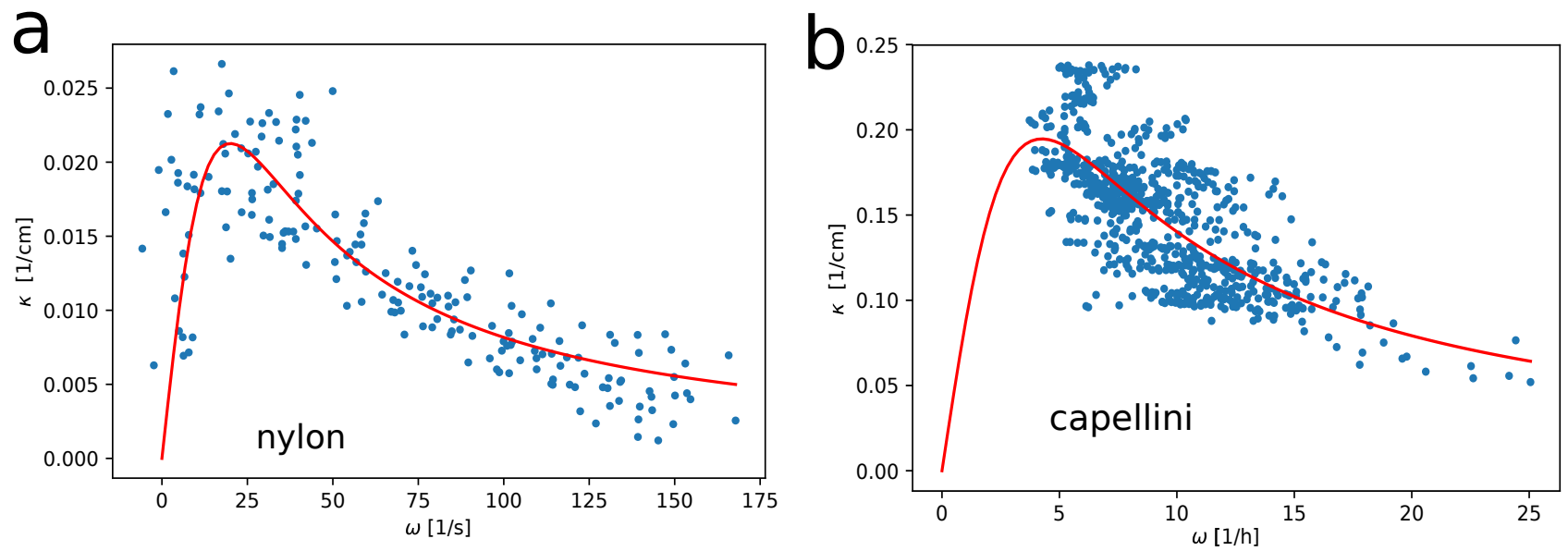

Fig. 4 Master curves, as described by Eq. 15, of curvature $\kappa$ vs. angular velocity $\omega$ for nylon fibers on a heated plate (a), and thin spaghetti (capellini) on a wet towel (b). Blue dots are experimental data obtained from both transient motion and of steady rolling fibers, the red curve is a fit to the theory, allowing to extract values for the propellant pumping rates and the propellant relaxation rates: one obtains $p=0.85$ [ \pm 0.04 (95\% confidence interval)] $\mathrm{s}^{-1}$ and $r=20.0[ \pm 1.0] \mathrm{s}^{-1}$ for nylon (diameter $0.4 \mathrm{~mm}$, temperature $160^{\circ} \mathrm{C}$ ) and $p=1.66[ \pm 0.02] \mathrm{h}^{-1}$ and $r=4.25[ \pm 0.12] \mathrm{h}^{-1}$ for capellini (diameter $1 \mathrm{~mm}$, humidity $60 \%$, temperature $25^{\circ} \mathrm{C}$.)

master curve, with low mobility values $\mu$ corresponding to the ascending branch and higher mobilities to the descending branch of the master curve.

The experimentally observed variance along the master curve could be partially rationalized by the inherent disorder of each sample (especially pronounced for the starch-based noodles used) and the lack of end-piece confinement of the filaments. The latter sometimes leads to a visible "hinging"-type of movement with a pronounced anti-phase oscillation of velocity and curvature (see also Fig. 7p). This behavior can be interpreted in terms of a time-

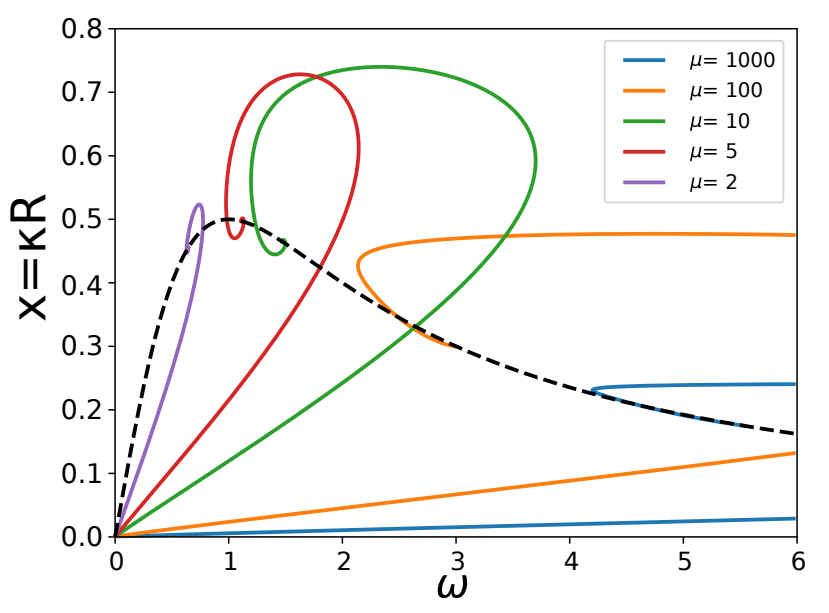

Fig. 5 Shown are numerically obtained trajectories from Eqs. $7 / 9$ in the plane in-plane eigenstrain $x(t)$ vs. angular velocity $\omega(t)$ and the corresponding master curve for the stationary state. All trajectories were initiated with zero strain (and hence curvature) and velocity, $x(0)=0$, $\omega(0)=0$. After a transient excursion they all land on the master curve, with low mobility values $\mu$ corresponding to the ascending branch and higher mobilities to the descending branch of the master curve. The black dashed line is the master curve given by Eq. 15 with $x=\kappa R$. Parameters: $r=p=1$. dependent mobility $\mu(t)$ that oscillates during one rotation cycle. Furthermore, inhomogeneities of the (here rigid) substrate surface can further contribute to variations in $\mu$. Both effects combined can lead to a "migration" along the $\kappa-\omega$ master curve for each sample.

However, in addition to such intrinsic disorder effects, there are more systematic reasons for this behavior necessitating extensions of the minimal model, discussed in the next section.

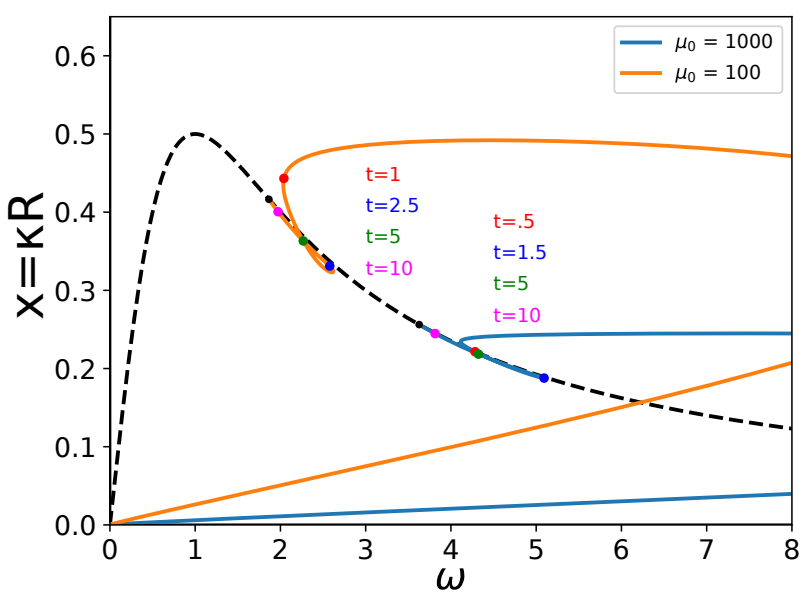

Fig. 6 Shown are numerically obtained trajectories from Eqs. 79, as in Fig. 5 but accounting for the dynamics of the storage mode, Eq. 16. We assumed $\mu=\mu_{0}(1-0.2 \bar{\psi})$ and $r_{\psi}=p_{\psi}=0.3$. Hence the time scale for the dynamics of the storage mode is slower than the $x, z$-modes by roughly a factor of three, and the Young's modulus (and hence the mobility) is reduced due the increased temperature to $20 \%$ of its initial value (at room temperature). The black dashed line is the master curve given by Eq. 15 with $x=\kappa R$. One can see that for both examples, after a rapid transient with high frequency and curvature excursions, the system stays close to the master curve for a substantial amount of time and moves in a nonmonotonic fashion (see indicated times as colored dots). 

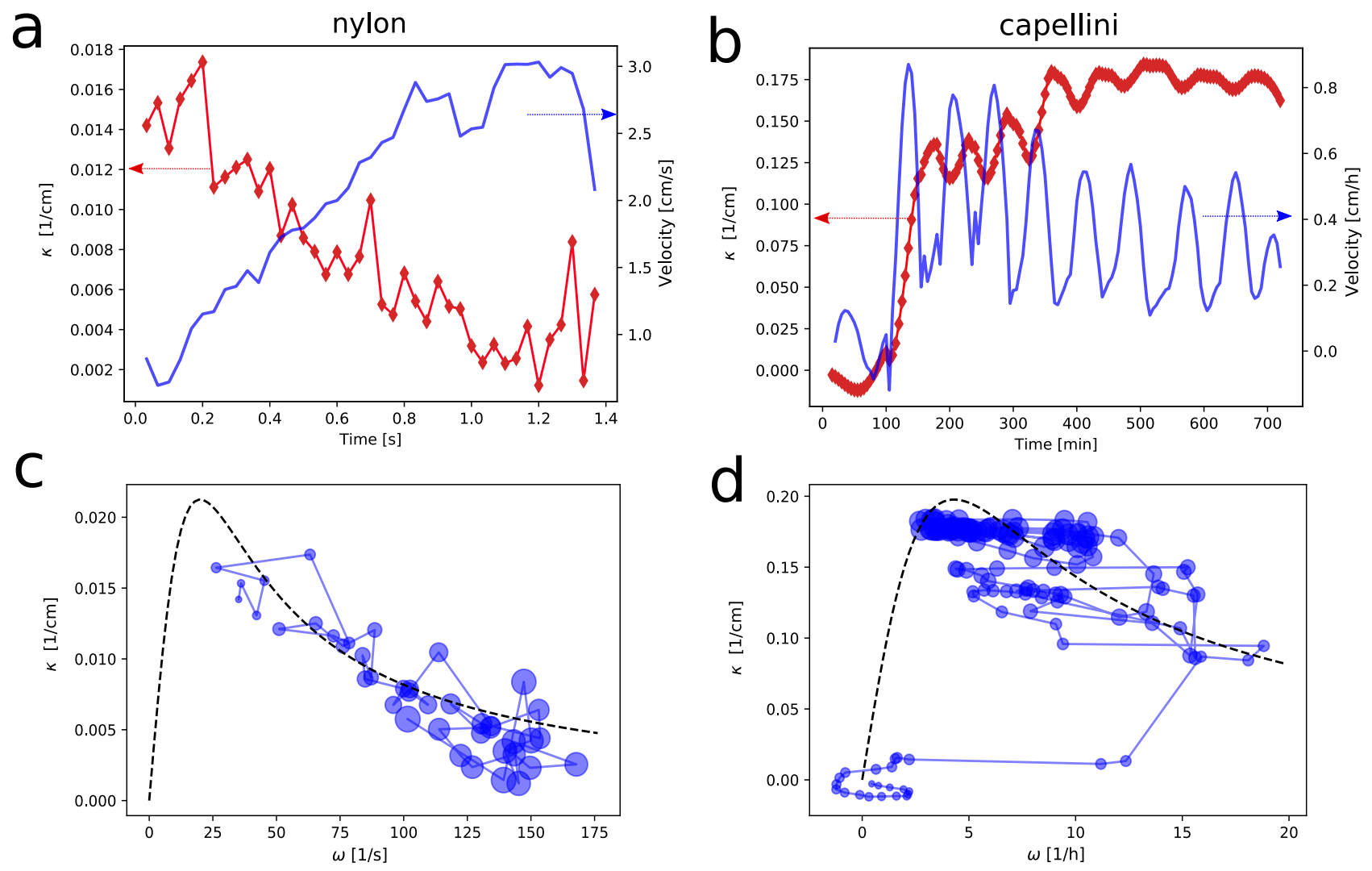

Fig. 7 a), b) Time dependence of fiber curvature (red) and translational velocity (blue) close to the onset of motion of fiberboids. Part a) shows representative data for a thermally driven nylon fiber and b) for a humidity-driven spaghetti, respectively. One can see that details are very different, especially there are transient oscillations for the noodles. Overall there is a certain anti-correlation between curvature and velocity, meaning the transients correspond to the descending branches of the respective master curves. This is evident when plotting the same data in the plane curvature vs. angular frequency as shown in parts $\mathrm{c}$ ) and d), where the dashed curves are the respective master curves.

\subsection{Refined relaxation models}

For the sake of simplicity, in the minimal model we have neglected certain processes and materials behavior. First, besides $x$ and $z$, there is a third dynamic "mode" that can become important: namely, a storage or internal reservoir mode, related to the average amount of propellant density, $\bar{\psi}$. The presence of this mode can be visualized experimentally by placing a hot fiber (formerly rolling on a heated plate) on a room temperature cold plate ${ }^{8}$. The fiber will still roll transiently - until it has equilibrated with the plate - but now curves oppositely with respect to the direction of motion as before, see supplementary movie 3 . The reason is that, now, heat is flowing out of the fiber.

A second effect concerns the material properties of the fiber. In case of thermal driving, the storage mode corresponds to the average temperature in the fiber cross-section and as such will affect the fiber's mechanical properties: most importantly, the bending rigidity, determined by Young's modulus $Y$, will vary with the average temperature (or the average density of propellant in the general case). In addition, other material parameters may also vary, for instance the (linear) thermal expansion coefficient depends on the reference temperature.

The storage mode, that was neglected in Ref. ${ }^{8}$, can be included in the model on a generic level, as well as directly derived from the underlying thermal diffusion-advection equation, see Appendix B. Its dynamics is given by

$$
\dot{\bar{\psi}}=-r_{\psi} \bar{\psi}+p_{\psi},
$$

hence analogously to the $z$-mode, the storage mode is pumped and relaxes, albeit with different (but related) rates $r_{\psi}, p_{\psi}$. Eqs. (7) and (9) remain unchanged, but now the mobility $\mu=$ $\mu(\bar{\psi})$ is a function of $\bar{\psi}$, stemming from the $\psi$-dependence of Young's modulus $Y(\bar{\psi})$. The main effect of the storage mode is exemplified in Fig. 6, where we assumed that $\bar{\psi}$ relaxes slower than $x, z$ and leads to a substantial softening of the Young's modulus. One can see that the system stays close to the master curve for a substantial amount of time while moving along it in a nonmonotonic fashion (see colored dots).

Yet another complexity stems from the fact that the propellant transfer is typically non-symmetric. This effect is especially pronounced when there is one solid-solid and one solid-air interface on the two sides of the fiber, giving rise to vastly different boundary conditions on the flux: considering thermal flux, typically, the conductive heat flux at the solid-solid interface is much faster than the diffusion-advection dominated flux at the solid-air interface. In that case it can be shown (see Appendix C) that Eq. (8) gets an additional term, coupling directly to the storage mode dynamics,

$$
\dot{z}=-r z+\omega x+p+\chi\left(-r_{\psi} \bar{\psi}+p_{\psi}\right)
$$


with $\chi$ a coupling coefficient, see appendix for details.

In case of a symmetric propellant transfer, i.e. perfect thermal contact at bottom and top with the boundary condition $T_{\text {ext|bottom }}=T_{\text {solid }}$ and $T_{\text {ext } \mid \text { top }}=T_{\text {air }}$, it can be shown by solving the rotationally advected heat equation in the fiber crosssection (see appendix B) that $\chi=0$. In this simplest case, the mean propellant density, $\bar{\psi}$, influences the dynamics only via slow changes in the mechanical parameters, as described above. In contrast, if a more realistic boundary condition is employed, namely $l_{t h} \frac{d}{d \rho} T+\left(T-T_{\text {ext }}\right)=0$ accounting for convective heat transport in the surrounding liquid (in our case air), $\chi$ has the following properties, see appendix C: $\chi$ is (i) finite, (ii) proportional to the thermal expansion coefficient $\alpha$ and (iii) increasing with the thermal length scale $l_{t h}$, until saturating for large values of $l_{t h} / R$.

Hence there are two important slow modes, not present in the minimal model: first, the dynamics of $\bar{\psi}$, which is slower than the one of the modes $x$ and $z$ (for geometrical reasons, $r_{\psi}<r$ holds), influences the dynamics via slow changes in the mechanical parameters. And second, in the general, asymmetric flux case, $\bar{\psi}$ also induces a slow variation of the effective driving of the $z$ mode like $p_{\text {eff }}(t)=p+\chi\left(-r_{\psi} \bar{\psi}(t)+p_{\psi}\right)$, cf. Eq. (17). Inspecting Eq. $(16)$, as $\bar{\psi}$ is equilibrating, $p_{\text {eff }}(t)$ exponentially relaxes towards $p$.

When placing a nylon fiberboid having room temperature on a heated plate, one hence expects the following slow relaxations in time of the parameters: the thermal expansion of nylon is typically non-linearly increasing ${ }^{8}$ in the relevant region $T \in[100-180]^{\circ} \mathrm{C}$, meaning it slowly increases with the mean temperature $\bar{\psi}$ until it reaches its stationary value. Hence the effective pumping rate of the $z$-mode, $p_{e f f}(t)$, increases due to its proportionality in the expansion coefficient $\alpha$. However, as the driving flux is asymmetric, at the same time the pumping rate relaxes due to equilibration of $p_{\text {eff }}$ towards $p$. Altogether, the behavior of the pumping rate is hence non-monotonous in time in the general case. In contrast, the second material coefficient affected by the storage mode, the mobility $\mu$, always decreases in time: it is proportional to Young's modulus $Y$, which softens with temperature.

Fig(7), d) show the time dependence of curvature (red) and rolling velocity (blue) for a thermally driven nylon and a humidity-driven spaghetti fiberboid, respectively. In both cases, one can observe an anti-correlation between curvature and velocity, i.e. the shown transient events close to the onset of motion are located on the descending branches of the respective master curves shown in Fig 4a),b). Interestingly, for nylon curvature decreases while velocity increases while noodles show the opposite trend, including oscillations related to the less strict confinement of the fiber ends.

\subsection{External forces and torque-velocity relation}

Up to now we have modeled fiberboids in uniform external environments and in absence of forces. To understand how they interact with external fields, substrate inhomogeneities or obstacles we can generalize the minimal model from Section 3.1 to

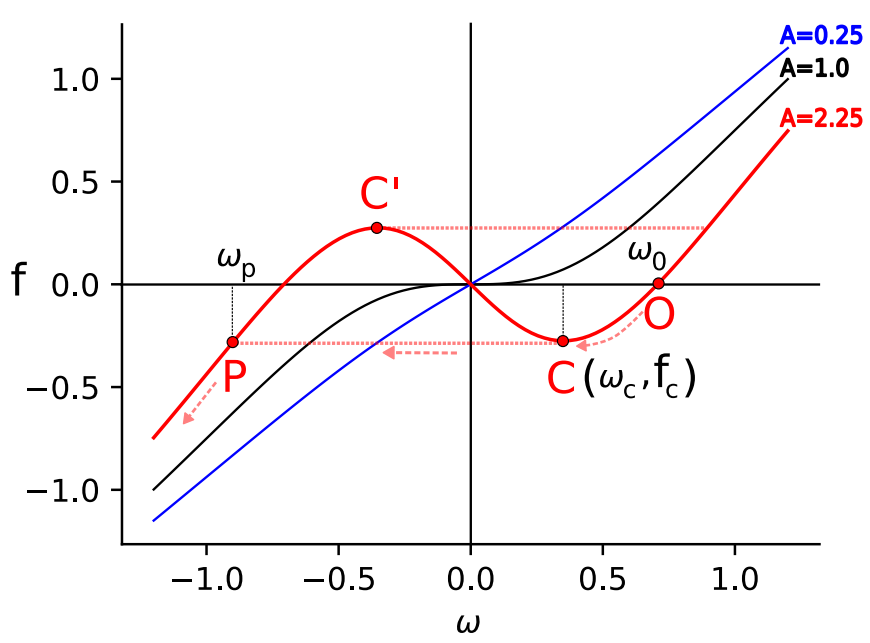

Fig. 8 The "motor relation" curves of fiberboids: scaled torque $f$ vs. angular velocity $\omega$ (in units of $r$ ), as given by Eq. 21) for selected values of the activity parameter $A(\mu=1, r=1, p=0.5,1,1.5)$. For large activity $A>1$ beyond unity (red curve) the motor curve has a typical hysteresis loop. Starting from zero torque $f=0$ (point $\mathrm{O}$ ), increasing the magnitude of the opposing torque $(f<0)$ we reach the critical point $\mathrm{C}$ with a critical torque $f_{c}$. Beyond $f_{c}$ the motion abruptly inverts direction and $\omega$ drops to a large negative value $\omega_{P}<0$ following now the direction of the driving torque (point $\mathrm{P}$ and further left to it). The branch $C C^{\prime}$ is unstable.

motion in an external potential $V(\phi)$ acting on the cross-section rotation angle $\phi(t)=\int \omega d t=S / R$ (with $S$ the distance traveled on the substrate). Substituting $\omega=\dot{\phi}$ in Eq. (7) and Eq. (8) and adding an additional torque $V^{\prime}(\phi)$ to Eq. (9) we obtain

$$
\begin{aligned}
\dot{x} & =-r x-\dot{\phi} z \\
\dot{z} & =-r z+\dot{\phi} x+p \\
\dot{\phi} & =-\mu x z+f(\phi)
\end{aligned}
$$

with $f(\phi)=-V^{\prime}(\phi) / \xi$.

Torque-angular velocity relation. Let us consider the simplest case where the external potential has a uniform gradient giving rise to a constant torque (scaled by friction) $f=$ const. In this case we can consider the fiberboid as an engine working against the constant external load $f$. In the steady-state limit (i.e. $\dot{\phi}=$ $\omega$ a constant, $\dot{x}=\dot{z}=0$ ) it is easy to derive the (torque-angular velocity) "motor-relation" for fiberboids:

$$
f(\omega)=\left[1-A\left(1+\frac{\omega^{2}}{r^{2}}\right)^{-2}\right] \cdot \omega
$$

with the reoccurring activity parameter $A=\mu p^{2} / r^{3}$ introduced earlier in Eq. 13 for the torque-free case. Depending on the magnitude of $A$, the torque-velocity curve can have one of two characteristic shapes, see Fig. 8. In the "weakly active" case, $A<1$, for which autonomous motility is absent at vanishing torque, the motor-relation is monotonic, with the forcing and motion always pointing in the same direction. In this case the motor exhibits a merely dissipative response with an effective internal friction $\xi_{\text {eff }} \propto f(\omega) / \omega$ that is positive, similar to the behavior of a fully passive object under the action of a torque. 
In the more interesting "strongly active" case, $A>1$, a nonmonotonicity arises and for $-\omega_{c}<\omega<\omega_{c}$ the motor curve has negative slope. In this case the system exhibits effectively a negative internal friction and the motor can roll against the torque. This opposition to the external torque is only possible as long as the motor is not overloaded, i.e. for $|f|<f_{c}$ below a critical torque $f_{c}$. Surpassing this torque, the motor inverts direction and runs with the torque. Close to the onset of motion, for $A \gtrsim 1$ just above its threshold value, $\omega$ is small. This allows us to expand Eq. 21 and imposing $\partial f / \partial \omega=0$ one obtains an estimate for the critical torque for $A \approx 1$ :

$$
f_{c} \approx-\frac{2}{3 \sqrt{6}} r(A-1)^{3 / 2}\left(\text { for } A \rightarrow 1^{+}\right) .
$$

Along similar lines in the opposite limit of large $A$ we have

$$
f_{c} \approx-\frac{3 \sqrt{3}}{16} r A(\text { for } A \rightarrow \infty)
$$

The results are intuitive: a more strongly driven system, with larger $A$, has a larger magnitude of critical torque. At the threshold $(A=1)$ the critical torque vanishes as the fiberboid is insufficiently driven and cannot bear any opposing load.
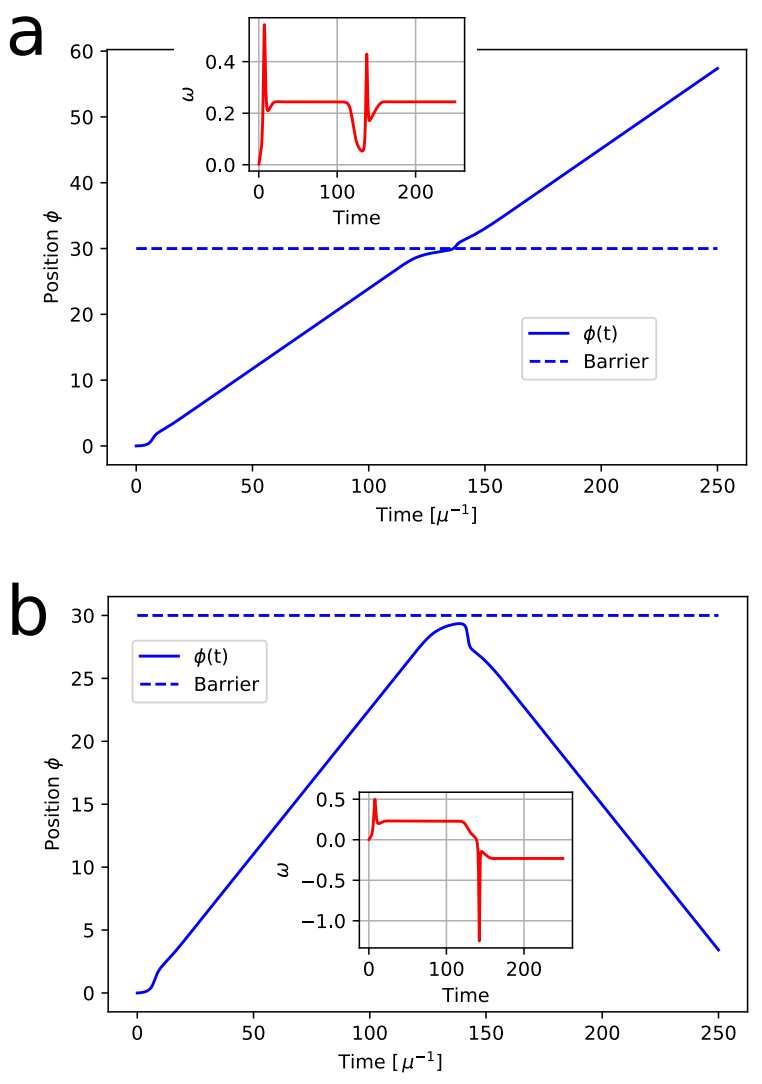

Fig. 9 The dynamics of fiberboids interacting with an obstacle (dashed line) as given by the dynamical Eqs. 1820 and the potential, Eq. 24, with $V_{0} / s=1, \mu=1$ and $r=0.1$. Shown is the rolling angle (traveled distance/fiber radius) as a function of time. For supercritical driving ( $p=$ $0.22, A=48.4$ ) the fiberboid overcomes the obstacle, see a), while for subcritical driving $(p=0.20, A=40)$ it bounces off the obstacle with a pronounced spike in angular velocity as shown in panel b). Insets in a) and b) show the angular velocities (in units of $\mu$ ).
Step barrier. A second interesting case is that of a localized obstacle. One can model an external obstacle at a position $\phi_{0}$ by a simple, smoothened step potential of the form

$$
V(\phi)=V_{0} \tanh \frac{\phi-\phi_{0}}{s}
$$

with the barrier height $V_{0}$ and $s$ a characteristic interaction range.

Fiberboids hitting such an obstacle can behave in two characteristic ways: For strong drive (i.e. A large) and a weak obstacle (i.e. small $V_{0} / s$ ), its angular velocity is only locally perturbed by the obstacle and the fiberboid penetrates this "barrier" continuing in the same direction, see Fig. 9a). In the regime of weak driving (small activity $A$ ) and sharper potentials (large $V_{0} / s$ ), however, the obstacle becomes effectively impenetrable and reflects the fiberboid, as shown in Fig.9p).

Interestingly, in such a dynamic scenario of engaging a localized obstacle the fiberboids can be forced to invert direction by torques $|f|<\left|f_{c}\right|$ that are even below their steady-state critical value. In Fig. 9p) and b), where the activities are $A=48.4$ and $A=40$, respectively, the predicted critical torques (from Eq. 23) are $f_{c}=-1.3$ and $f_{c}=-1.6$. Although the maximal torque exerted by the obstacle is in both cases $f=-1$, only the stronger driven fiberboid (the one in Fig. 9a) can dynamically penetrate the barrier.

Another interesting observation is that a reflection can happen with transiently highly elevated speeds, well in excess the steady state angular velocity. Such an accelerated bounce-off relaxation behavior is also observed experimentally, see the following Section and Fig. 10p). It can be rationalized by the observation that the magnitude of the steady-state angular velocity is always larger after the directional switching event than before it, $\left|\omega_{p}\right|>\left|\omega_{c}\right|$ (see the jump from point $\mathrm{C}$ to $\mathrm{P}$ in Fig. 3). The torque assists (hinders) the motion after (before) the direction inversion.

\section{4 "Social" behavior: Interaction with walls and other fiberboids}

As we have seen, fiberboids are bidirectional and display spontaneous symmetry breaking at the onset of motion. In the previous section we also proposed a phenomenological model for fiberboids bouncing into obstacles. Both suggests that fiberboids should be able to respond to their environment, e.g. by inverting their direction of motion, and that they are poised to engage in interesting collective behavior, see supplementary movie 4 . We now study experimentally the two-body behavior and answer the questions: What happens when fiberboids engage immovable obstacles or when they they frontally collide with each other?

The answer to this question depends on whether the fiberboids are "vexers" or "cavers", cf. Fig. 2p), i.e. if they curve towards or away from their direction of motion. In general, by simple geometric reasoning, cavers (like thermally driven nylon) establish single-point contact at their center apex position, while vexers (like humidity-driven noodles) make contact at their distant end points. The contact in the former case is hence much more stable and leads to more predictable interactions. In the latter case, the two-point contact renders the interaction less predictable because the ends are often insufficiently confined to the plane. 

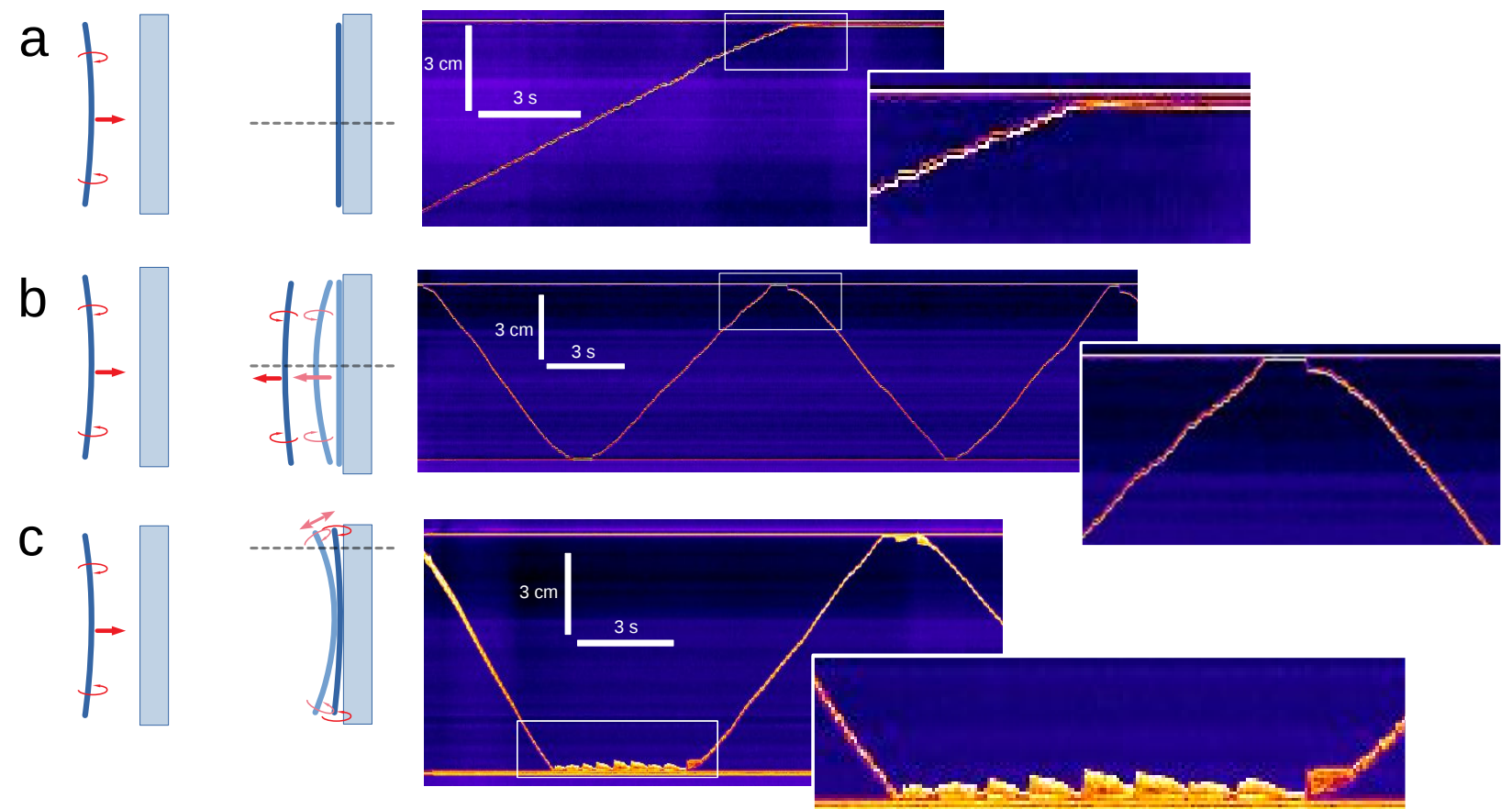

Fig. 10 Typical outcomes for thermally driven nylon fiberboids hitting obstacles, implemented via glass slides lying on the heated plate. Panel a) shows the case for low driving rate $\left(T=130^{\circ} \mathrm{C}\right)$, where the fiber gets stalled at the wall and completely stops rolling. Panel b) shows that for intermediate driving $\left(T=160^{\circ} \mathrm{C}\right)$ the fiber is effectively reflected from the walls, in a nicely reproducible manner (the kymograph shows 4 reflection events). Note the spike in velocity immediately after bounce-off. Panel c) shows that for too strong driving $\left(T=170^{\circ} \mathrm{C}\right)$, the fiberboid gets captured at the wall, but continues rolling against it, performing a kind of flapping, cf. the zoomed-in kymograph. The horizontal dashed lines in the sketches on the left indicate the position of the cross-section tracked in the respective kymographs on the right. See supplementary movie 5.

\subsection{Cavers}

Fig. 10 shows the generic scenarios found for thermally driven fiberboids of "caver"-type hitting a wall. Experimentally, this can be simply studied by confining the rolling fiber between two glass slides (placed as 2D obstacles) on the heating plate. Panel a) shows a weakly driven fiber $\left(T=130^{\circ} \mathrm{C}\right)$ that is simply stopped by the wall. The reason is a combination of increased friction due to the presence of the wall and unfavorable in-plane heat transfer through the glass wall to the fiber. In fact, the back of a rolling nylon fiber is warmer than the front (cf. Fig. 22); for thermally driven fibers the color-code there means red $=$ hot, blue $=$ cold). But the front receives additional thermal energy from the glass wall, disfavoring the motion.

However, if the temperature is further increased, as shown in Fig. $10 \mathrm{p}$ ) for $T=160^{\circ} \mathrm{C}$, the fiber gets effectively reflected from the wall: the middle part of the fiber is slowing down and "loosing" curvature just to an extent that the fiber ends are able to catch up and the thermal transfer through the glass can invert the direction of motion. As seen in the same figure, reflections are nicely reproducable at both walls and they are often accompanied by jumps in velocity, cf. Fig. 9p). For even higher driving, cf. Fig. 10.), the fiber stubbornly and continuously rolls against the wall, while its ends, not in direct contact to the wall, perform a kind of flapping motion.

At the contact with the wall several effects play a role making a quantitative theory very difficult. (1) First, there is the oppos- ing force exerted by the wall. (2) Second, there is a loss of the rolling contact (including slip at the bent sides of the fiber) and in addition an increased friction at the wall. (3) The propellant (heat) transfer at the wall is severely altered: the glass slides have similar temperature as the heated surface, which should give rise to an additional pumping term (similar to $p$ ) in the equation for the planar strain $x$. In addition, the cooling effect of air convection is altered by the presence of the wall (cf. the discussion of propellant flux asymmetry and the thermal length scale $l_{t h}$ in appendix C). Finally, due to curving, it is no longer possible to treat the cross-sections along the fiber as equal - some are in contact to the wall while others, especially the ends, are not.

Nevertheless, the models developed in section 3 can account phenomenologically for most of the generic aspects of fiberboids colliding with walls (or with other fiberboids). In fact, the case shown in Fig. 10a) corresponds to weak driving (small $p$ but $A=$ $\frac{\mu p^{2}}{r^{3}}>1$ in the absence of the wall) and a rather rough obstacle whose effect can be interpreted by a lowering of the mobility $\mu$ upon contact as a combination of effects (1) and (2) discussed above. The fiberboid stops since the first term in Eq. 11 decreases with the reduced $\mu$ resulting from wall friction: $A$ becomes less than one and rolling is no longer possible.

The case of Fig. 10p) corresponds to intermediate $p$, hence $A$ is larger and the reduction of mobility upon contact does not reduce $A$ below one. Consequently, a finite rotation speed is still possible. However, Eq. 12 implies that the curvature is still reduced due to 
friction: the first, positive contribution scales like $\mu^{-1 / 2}$ while the second, negative contribution as $\mu^{-1}$. This leads to the catchup of the ends described above and then the fiber is ready to invert due to the additional heat influx from the wall, cf. point (3) above, into what will become the new back of the reversed fiber. If $p$ is even larger, the reduction in $\mu$ is not sufficient to reverse the fiber and rolling against the wall persists, corresponding to the case shown in Fig. 10.).

The cases shown in Fig. 10 can be also interpreted using the model including an external potential as described in section 3.3 . Here the potential models the effects (1) and (2) from above on a phenomenological level. Case b) corresponds to the case where the driving is intermediate, as shown in Fig. 9p). The forcevelocity relation in addition nicely explains the pronounced spike in velocity seen immediately after the inversion of the fiber, see Fig. 10p). Lower driving corresponds to stopping, while larger drive to continuing rolling without inversion, where however the fiberboid cannot penetrate the step potential at all. It is likely that the complete stopping at the wall is furthermore strongly affected by additional effects, here not taken into account, like the critical threshold for the onset of motion under dry friction.

For binary collisions between two fiberboids, quite similar effects to wall collisions are observed. Fig. 11 shows the main types of events for binary central collisions between two thermally driven fiberboids of equal lengths. Panels a),b) show what we call "follower-leader" behavior: for a short transient, both fibers roll against each other, but then the "weaker" inverts direction and the pair now travels together. This pairing is quite robust against further collisions or perturbations. Panels c),d) shows what we call "dancing pair" behavior: here, the pair keeps on rolling against each other for long times. At the same time, the common center of mass goes back and forth, due to flapping-type curvature variations.

\subsection{Vexers}

Vexers, like the hygroscopically driven spaghetti, can exhibit similar "social behavior" as cavers, as exemplified in Fig. 12a) by an inversion event upon a collision. However, unlike cavers, vexer-type fiberboids exhibit more random, generically unstable, multi-point contact configurations. This leads more frequently to complex out-of-plane deformations, followed by mutual entanglement or amusing "tunneling" behavior where one filament lifts and bypasses the other, as exemplified in Fig. 12p). Such richness and variability of collision outcomes makes vexers overall less suitable for investigating their collective behavior.

\section{Conclusions and Outlook}

Fiberboids are a new member of the class of active, self-propelled particles ${ }^{2[16}$ that was surprisingly long hiding in the plain view, before being only recently described 8 .

Fiberboids naturally share some similarities with other actively rolling systems, like Quincke spinners ${ }^{3}$, Marangoni-driven droplets ${ }^{6}$ or Leidenfrost droplets ${ }^{7 / 17}$. However, in contrast to those they do not rely on fluid motion. They do develop a continuously propagating internally rotating mode akin to Bénard or
Marangoni convection rolls in fluids, yet surprisingly this mode is an elastic prestress "flowing" through a solid material ${ }^{8}$. This naturally has several advantages: the self-propelled particle is not eaten-up by the propulsion process itself (as for Leidenfrost and Marangoni driving) and it can result, especially for the thermal drive, in a constant steady-state velocity.

Another advantage of the fiberboid mechanism is its practical simplicity: Many everyday objects can be easily turned into fiberboids, as demonstrated here: pieces of fishing line spin quickly on a hot plate, while spaghetti noodles spin both rapidly on a hot plate ${ }^{8}$, as well as much more slowly on wet cloth. The latter example also shows that different fluxes can be employed to drive fiberboids, and on vastly different timescales (differing by orders of magnitudes). Last but not least, fiberboids are anisotropic objects and hence should display interesting new collective effects beyond the existing spherical rollers 3 [6/7|17.

Here we have laid out the basic phenomenology and proposed generic models of increasing complexity for the dynamics of fiberboids. We started from a simple two-mode model, describing the dynamics of the propellant gradients in two orthogonal directions of the fiber cross section. This simple model phenomenologically captures many of the features of fiberboid behavior, including the symmetry breaking at the bifurcation point and a curvature-velocity dynamics following a simple master curve. To treat all the experimentally observed phenomena, including dynamic transients, we extended the two-mode description and introduced a third degree of freedom - a "storage mode" corresponding to the mean propellant density in the cross section. Interestingly, this mode couples in several ways, namely both via the mechanics and the dynamics, to the other modes.

By studying fiberboid collision events, both with walls and other fiberboids of the same type, we have extracted the conceptual ingredients for future studies of the collective behavior of (many) fiberboids. Although many of the generic aspects of the fiberboid collision events could be rationalized by the model, others require future model sophistication and extensions. Purely 1D systems of interacting active particles have been studied for Marangoni droplets ${ }^{18}$ and crawling cells ${ }^{19}$ in confined spaces. These also showed both velocity inversion and follower-leader behavior and, accordingly, complex collective behavior. Fiberboids add another, easy to study, table-top example to this generic class of phenomena able of unlocking the charms of non-equilibrium physics to undergraduate and high-school students, as we find from own experience.

We conclude with a simple message: fiberboids should be a general phenomenon in nature, as they require only a few physical ingredients, which are: a) An interface between two phases A and $\mathrm{B}$, at least one of which is a fluid (to allow for mobility). b) The phases A and B should be out-of-equilibrium, giving rise to a flux of energy/matter across their interface. c) Straight filaments, with cylindrical cross-section, that are pinned (confined) at the interface and that mechanically respond to the flux.

These three conditions are indeed generic, also on the microscopic scale, as we are surrounded by interfaces that frequently are out-of-equilibrium. Naturally ordered, biological fibers can be trapped there by Van der Waals or electrostatic forces or Picker- 

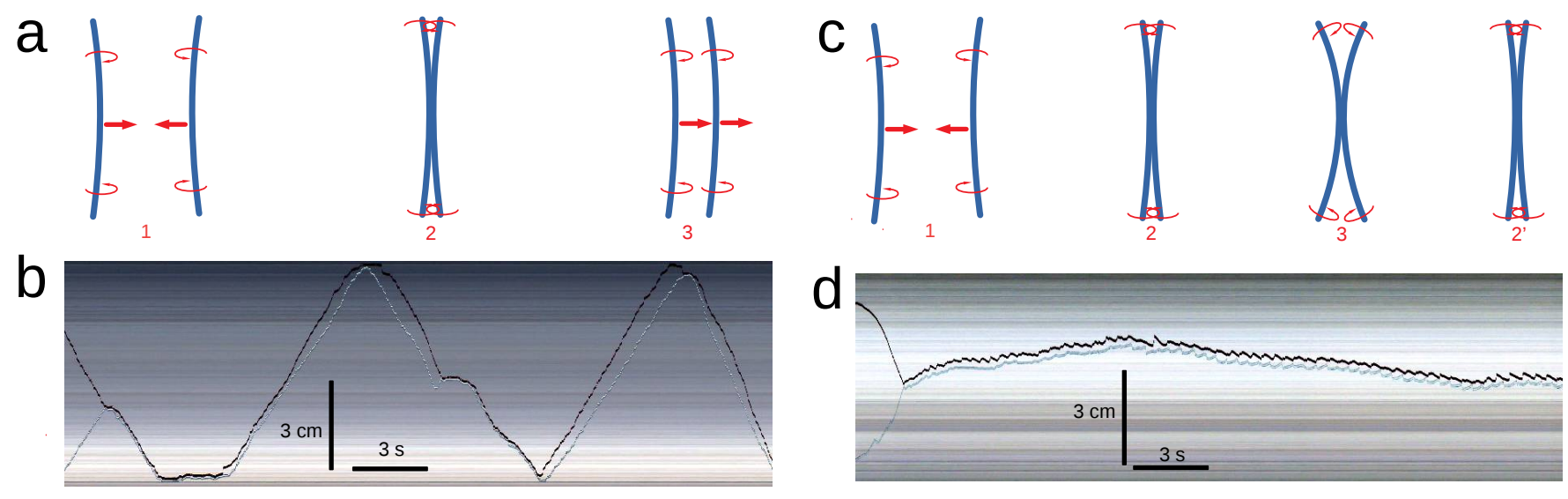

Fig. 11 Typical binary collision events of thermally driven nylon fiberboids of equal length. Two main outcomes for the head-on collision dominate: panel a) sketches an event, where after rolling against each other for a short transient time, one fiber switches directionality such that finally both move together as a pair with almost equal velocity. This can be seen in the kymograph shown in b) at the very left. Note that the kymograph also displays several collisions of the fiberboid pair with boundaries, where the pair is effectively reflected. Hence this "follower-leader" mode is quite robust against perturbations. Panel c) sketches a second common event, where the pair keeps on rolling against each other, with the center of mass of the "dancing pair" slightly going back and forth due to curvature variations ("flapping"). As can be seen from the time scale bar in d), this state can be stable for a substantial time (minutes).

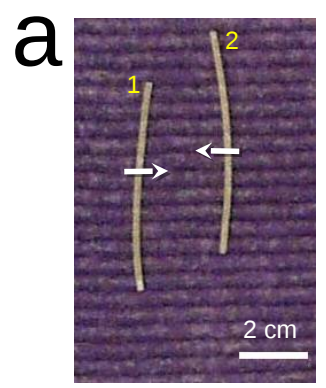

$\mathrm{Oh}$

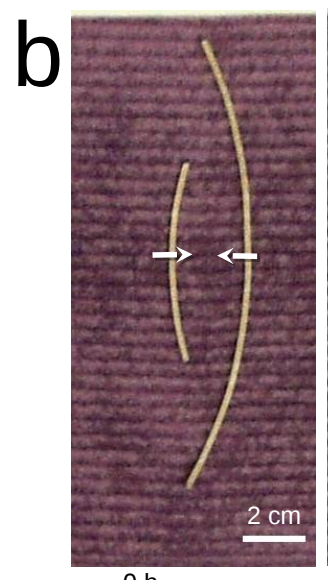

$\mathrm{Oh}$

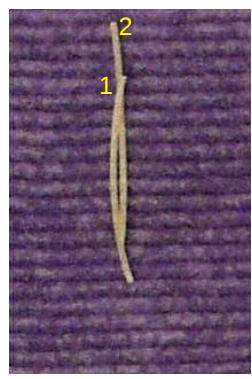

$1.5 \mathrm{~h}$

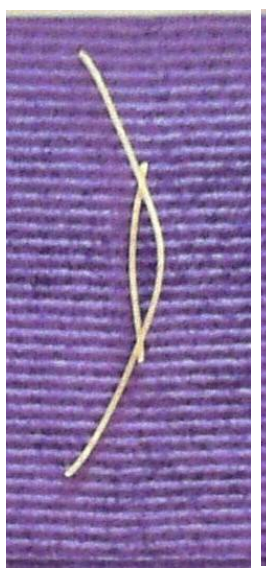

$7 \mathrm{~h}$

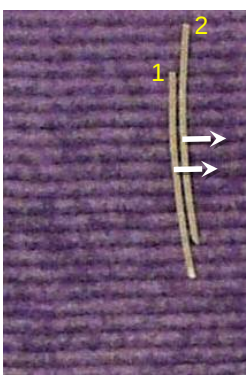

$4 \mathrm{~h}$

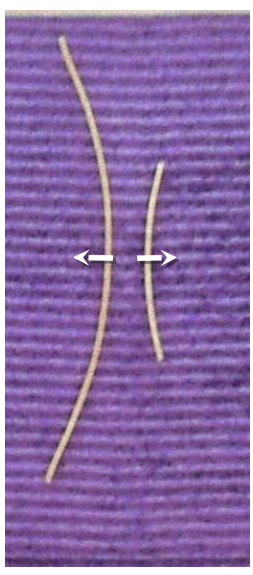

$10 \mathrm{~h}$
Fig. 12 Typical binary collision events for vexers, realized by spaghetti on a wet towel. Panel a) shows a collision and inversion of short spaghetti,See supplementary movie $6, b)$ a collision of one long and one short spaghetti. In the latter case, the longer specimen transiently leaves the planar confinement and "jumps" over the shorter one. See supplementary movie 7 . ing pinning, and driven by a multitude of chemical and thermodynamic fluxes. Moreover, it has been shown that many biofilaments, like microtubules ${ }^{20 \mid 21}$ and intermediate filaments ${ }^{22}$ can spontaneously break their cylindrical symmetry and self-buckle. This phenomenon happens under internal prestress and leads to symmetry broken zero energy ("wobbling") modes. The latter zero modes are similar to the dissipatively emerging (rotating) curvature mode in fiberboids ${ }^{8}$ and should further facilitate the emergence of the fiberboid effect by eliminating the threshold for buckling. Related ideas of generating fluxes via various ratchet effects in circular DNA ${ }^{23}$ could further expand our toolbox to "motorize any filament".

Recently, we have speculated ${ }^{15}$ that filamentous viruses like ebola might utilize fiberboid physics to roll on surfaces. Considering the ease with which we generated fiberboids, in particular the motorization of spaghetti in various ways, it seems in fact unlikely that nature could have overlooked the motif.

\section{Appendix A: Driving torque}

Here we calculate the driving torque generated by the fiberboid mechanism using slender rod elasticity coupled to the propellant density which induces expansion/contraction according to Eq. (1).

Let the interface be the $X-Y$-plane. We define the lab frame by laying the fiber axis along $Y$ and consider a cross-section of the fiber with the fiber's (vectorial) curvature fixed along $X$, hence $\vec{\kappa} \cdot e_{x}=\kappa_{x}=\kappa$. We introduce the angle $\Phi$ with respect to the $X$ axis, describing a rotation of the fiber's cross-section along its axis (i.e. the $Y$-axis) and defining the co-rotating internal coordinate system $e_{1}=(\cos \Phi, \sin \Phi), e_{2}=(-\sin \Phi, \cos \Phi)$. We call these internal coordinates $\left(X^{\prime}, Z^{\prime}\right)$ or in polar form $(\rho, \phi)$. Obviously, they are related to the lab frame coordinates via a rotation matrix

$$
\left(\begin{array}{l}
X^{\prime} \\
Z^{\prime}
\end{array}\right)=\left(\begin{array}{cc}
\cos \Phi & \sin \Phi \\
-\sin \Phi & \cos \Phi
\end{array}\right)\left(\begin{array}{l}
X \\
Z
\end{array}\right)
$$


In these internal coordinates the axial strain in the cross-section $\left(\varepsilon_{Y Y}\right)$ for a slender rod is given by

$$
\varepsilon(\rho, \phi)=-\rho\left(\kappa_{1} \cos \phi+\kappa_{2} \sin \phi\right)+\bar{\varepsilon}-\varepsilon_{\psi}(\rho, \phi) .
$$

The first contribution is bending (with the negative sign indicating compression within the cross-section at the inwards curved side). $\bar{\varepsilon}$ is the strain related to axial compression/elongation and $\varepsilon_{\psi}(\rho, \phi)$ is the propellant-induced (axial) prestrain. Using that the curvatures in the rotating frame are given by $\kappa_{1}=\vec{\kappa} \cdot e_{1}=$ $\kappa \cos \Phi, \kappa_{2}=\vec{\kappa} \cdot e_{2}=-\kappa \sin \Phi$, one gets

$$
\varepsilon(\rho, \phi)=-\rho \kappa \cos (\phi+\Phi)+\bar{\varepsilon}-\varepsilon_{\psi}(\rho, \phi)
$$

The elastic energy of the considered cross-section (i.e. energy per length) reads $E=\frac{Y}{2} \int(\varepsilon(\rho, \phi))^{2} \rho d \rho d \phi$ with $Y$ Young's modulus. Inserting the strain yields three contributions

$$
\begin{aligned}
E(\kappa, \bar{\varepsilon}, \Phi)= & \frac{1}{2} B \kappa^{2}+\frac{Y}{2} \int\left(\bar{\varepsilon}-\varepsilon_{\psi}(\rho, \phi)\right)^{2} \rho d \rho d \phi \\
& +Y \kappa \int \cos (\phi+\Phi) \varepsilon_{\psi}(\rho, \phi) \rho^{2} d \rho d \phi .
\end{aligned}
$$

Clearly, the first term the standard bending energy with $B=\frac{\pi}{4} Y R^{4}$ the bending stiffness. To evaluate the others, one needs an expression for $\varepsilon_{\psi}$.

The propellant-induced (axial) prestrain, cf. Eq. (1), is given by $\varepsilon_{\psi}(\rho, \phi)=\alpha \psi(\rho, \phi)$, where complications such as shear in the cross-section, torsional contributions etc. are disregarded for simplicity. For the propellant distribution we make the following ansatz,

$$
\psi(\rho, \phi)=\Delta \psi_{c} \frac{\rho}{R} \cos \phi+\Delta \psi_{s} \frac{\rho}{R} \sin \phi+\tilde{\psi},
$$

introducing the two principal differences ('modes') in the rotating frame, $\Delta \psi_{c}$ and $\Delta \psi_{s}$, as well as the homogeneous contribution $\tilde{\psi}$.

We now assume that the compressive/elongational strain relaxes fast. Minimization of the energy, Eq. (28), w.r.t. $\bar{\varepsilon}$ yields

$$
\bar{\varepsilon}=\frac{1}{\pi R^{2}} \int \varepsilon_{\psi}(\rho, \phi) \rho d \rho d \phi .
$$

Insertion of the ansatz, Eq. (29), then simply yields $\bar{\varepsilon}=\alpha \tilde{\psi}$, meaning that the compressive/elongational strain just compensates the homogeneous part of the propellant-induced stress.

We use this to simplify the elastic energy, perform all integrals and get

$$
E(\kappa, \Phi)=B\left\{\frac{1}{2} \kappa^{2}+\alpha \frac{\kappa}{R}\left(\Delta \psi_{c} \cos \Phi-\Delta \psi_{s} \sin \Phi\right)\right\}
$$

plus an additional term that is curvature- and angle-independent and hence has no consequence for the dynamics.

Neglecting initial, transient reshaping events of the linear fiber, the stationary curvature $\kappa_{s}$ of the fiber can be obtained by minimization w.r.t. $\kappa$, yielding

$$
\kappa_{s}=-\frac{\alpha}{R}\left(\Delta \psi_{c} \cos \Phi-\Delta \psi_{s} \sin \Phi\right) .
$$

In turn, the driving torque, generating the rolling motion if it overcomes the prevalent dissipation mechanisms, is the conjugate quantity to the angle $\Phi$ and hence given by $m=-\frac{\partial E}{\partial \Phi}$, implying

$$
m=B \alpha \frac{\kappa}{R}\left(\Delta T_{c} \sin \Phi+\Delta T_{s} \cos \Phi\right) .
$$

Both expressions involve the modes $\Delta \psi_{c}$ and $\Delta \psi_{s}$ in the rotating frame. To express them in lab frame one uses the definition of polar coordinates and Eq. 25) in the ansatz, Eq. (29), to get

$\psi(\rho, \phi)-\tilde{\psi}=\left(\Delta \psi_{c} \cos \Phi-\Delta \psi_{s} \sin \Phi\right) \frac{X}{R}+\left(\Delta \psi_{c} \sin \Phi+\Delta \psi_{s} \cos \Phi\right) \frac{Z}{R}$

The two principal modes in the lab frame are hence identified as

$$
\begin{aligned}
& \Delta \psi_{x}=\Delta \psi_{c} \cos \Phi-\Delta \psi_{s} \sin \Phi=: \Delta_{\leftrightarrow} \psi, \\
& \Delta \psi_{z}=\Delta \psi_{c} \sin \Phi+\Delta \psi_{s} \cos \Phi=: \Delta_{\uparrow} \psi .
\end{aligned}
$$

This leads to the simple and instructive expressions

$$
\kappa_{s}=-\frac{\alpha}{R} \Delta_{\leftrightarrow} \psi, m=B \alpha \frac{\kappa_{s}}{R} \Delta_{\uparrow} \psi .
$$

I.e. the curvature is determined by the in-plane propellant gradient and the driving torque by both this curvature and the out-of plane propellant gradient. Putting both together and absorbing the expansion coefficients by introducing the eigenstrains yields

$$
m_{\text {drive }}=-\frac{B}{R^{2}}\left(\Delta_{\leftrightarrow} \varepsilon_{y y}^{e i g}\right)\left(\Delta_{\uparrow} \varepsilon_{y y}^{e i g}\right),
$$

hence the same result obtained by scaling arguments in Eq. (6)

\section{Appendix B: Symmetric thermal drive}

In the case of thermal driving, one has to solve the rotationally advected thermal diffusion equation

$$
\partial_{t} T=\frac{D}{R^{2}} \nabla^{2} T+\omega \partial_{\phi} T .
$$

Note that we rescaled space by the fiber radius $R$, such that in polar coordinates, $T(\rho, \phi), \rho \in[0,1]$. For the simplest case of symmetric thermal contact, the boundary condition (BC) at the cylinder surface reads

$$
T(1, \phi)-T_{\text {ext }}(1, \phi)=0
$$

To capture the essential features, this $\mathrm{BC}$ is approximated by

$$
T_{\text {ext }}(1, \phi)=\frac{T_{s}-T_{\text {air }}}{2} \cos \phi+\frac{T_{s}+T_{\text {air }}}{2}=T^{-} \cos \phi+T^{+},
$$

Like that $T_{\text {ext }}(1,0)=T_{S}$ is the temperature at the bottom ('surface') and $T_{\text {ext }}(1, \pi)=T_{\text {air }}$ is the temperature at the top ('air').

We now are looking for a solution of the form

$$
T=C(\rho) \cos \phi+S(\rho) \sin \phi+\tilde{T}(\rho) .
$$

We will later identify the $z$-mode of the main text (as $C$ ), the $x$-mode (as $S$ ) and the storage mode $\bar{\psi}$ (as $\tilde{T}$ ), respectively. The symmetry-adapted functions to the problem are the socalled Zernike polynomials which have the general form $Z_{n}^{m}=$ $R_{n}^{m}(\rho) \cos (m \phi), Z_{n}^{-m}=R_{n}^{m}(\rho) \sin (m \phi)$. We will need only the first 
few which are given by

$$
\begin{aligned}
& Z_{1}^{1}=2 \rho \cos \phi, Z_{1}^{-1}=2 \rho \sin \phi, \\
& Z_{3}^{1}=\sqrt{8}\left(3 \rho^{3}-2 \rho\right) \cos \phi, Z_{3}^{-1}=\sqrt{8}\left(3 \rho^{3}-2 \rho\right) \sin \phi, \\
& Z_{0}^{0}=1, Z_{2}^{0}=\sqrt{3}\left(2 \rho^{2}-1\right) .
\end{aligned}
$$

Putting the ansatz Eq. (40) with

$$
C=c_{1} R_{1}^{1}+c_{2} R_{3}^{1}, S=s_{1} R_{1}^{-1}+s_{2} R_{3}^{-1}, \tilde{T}=T_{0} R_{0}^{0}+T_{2} R_{2}^{0}
$$

into the BC (39) and neglecting higher modes ( $m \geq 2$ ) yields the three conditions $C(1)-T^{-}=0, S(1)=0$ and $\tilde{T}(1)-T^{+}=0$, that can be solved for $c_{2}, s_{2}$ and $T_{2}$ to yield the following ansatz, fulfilling the BCs (up to $m=1$ ):

$$
\begin{aligned}
T= & s_{1}\left[Z_{1}^{-1}-\frac{1}{\sqrt{2}} Z_{3}^{-1}\right]+c_{1}\left[Z_{1}^{1}-\frac{1}{\sqrt{2}} Z_{3}^{1}\right]+\frac{T^{-}}{2 \sqrt{2}} Z_{3}^{1} \\
& +T_{0}\left[Z_{0}^{0}-\frac{1}{\sqrt{3}} Z_{2}^{0}\right]+\frac{1}{\sqrt{3}} T^{+} Z_{2}^{0}
\end{aligned}
$$

We now insert this ansatz into Eq. (37) and project on the different modes via $\int_{0}^{1} \ldots Z_{1}^{-1} \rho d \rho$ to project on $Z_{1}^{-1}$ (and analogously for $Z_{1}^{1}$ and $Z_{0}^{0}$ ). Using the relations for the Laplacian of the Zernike polynomials, $\Delta Z_{0}^{0}=0, \Delta Z_{1}^{1}=0, \Delta Z_{3}^{1}=24 Z_{1}^{1}, \Delta Z_{2}^{0}=8 Z_{0}^{0}$, as well as orthogonality, yields

$$
\begin{aligned}
& \dot{s}_{1}=-\frac{24 D}{\sqrt{2} R^{2}} s_{1}-\omega c_{1}, \\
& \dot{c}_{1}=-\frac{24 D}{\sqrt{2} R^{2}}\left(c_{1}-\frac{T^{-}}{2}\right)+\omega s_{1}, \\
& \dot{T}_{0}=-\frac{8 D}{\sqrt{3} R^{2}}\left(T_{0}-T^{+}\right) .
\end{aligned}
$$

Identifying $x=\alpha s_{1}, z=\alpha c_{1}$ and $\bar{\psi}=T_{0}$, this can be written as in the main text, i.e.

$$
\begin{aligned}
\dot{x} & =-r x-\omega z, \\
\dot{z} & =-r z+\omega x+p, \\
\dot{\bar{\psi}} & =-r_{\psi} \bar{\psi}+p_{\psi},
\end{aligned}
$$

with the rates

$$
r=\frac{24 D}{\sqrt{2} R^{2}}, p=\frac{24 D}{\sqrt{2} R^{2}} \frac{\alpha\left(T_{s}-T_{a i r}\right)}{4}
$$

for the $x, z$-modes (where $\alpha$ is the thermal expansion coefficient) and

$$
r_{\psi}=\frac{8 D}{\sqrt{3} R^{2}}, p_{\psi}=\frac{8 D}{\sqrt{3} R^{2}} \frac{T_{s}+T_{a i r}}{2},
$$

for the storage mode.

As it should be, the stationary state for the storage mode is $\bar{\psi}=\frac{p_{\psi}}{r_{\psi}}=\frac{T_{s}+T_{\text {air }}}{2}$, i.e. the mean temperature. The stationary heat conducting state is given by $z=\frac{p}{r}=\frac{\alpha\left(T_{s}-T_{a i r}\right)}{4}$, i.e. proportional to the mean temperature difference.

\section{Appendix C: Asymmetric thermal drive}

As discussed in the main text, a more realistic boundary condition is

$$
\frac{l(\phi)}{R} \frac{d}{d \rho} T(1, \phi)+\left[T(1, \phi)-T_{e x t}(1, \phi)\right]=0 .
$$

Here $l(\phi)$ is a thermal length scale describing convective transport in the fluid medium (air) at the top, see Ref. ${ }^{8}$. We assume $l(\phi=\pi)=l_{t h}$ at the top and at the bottom $l(\phi=0)=0$, i.e. still perfect thermal contact at the metal surface. The symmetryadapted approximated version of this BC then reads

$$
l(1, \phi)=(1-\cos \phi) \frac{l_{t h}}{2}
$$

and $T_{\text {ext }}(1, \phi)$ as defined in Eq. (39). Using the same Zernikemode expansion and projection yields the equations

$$
\begin{aligned}
\dot{x} & =-r x-\omega z, \\
\dot{z} & =-r z+p+\omega x+\chi\left(-r_{\psi} \bar{\psi}+p_{\psi}-\varepsilon z\right), \\
\dot{\bar{\psi}} & =-r_{\psi} \bar{\psi}+p_{\psi}-\varepsilon z .
\end{aligned}
$$

Now $r, p, r_{\psi}, p_{\psi}$ are all functions of $\lambda=\frac{l_{t h}}{R}$ that can be given explicitly. More importantly, a coupling between $z$ and $\bar{\psi}$ arises, with

$$
\varepsilon=\frac{8}{\sqrt{3}} \frac{D}{\alpha R^{2}} \frac{\frac{6 \lambda}{7 \lambda+2}}{1+\frac{3}{4} \lambda \frac{7 \lambda+4}{7 \lambda+2}}, \chi=\alpha \frac{9 \sqrt{3}}{2 \sqrt{2}} \frac{\lambda}{7 \lambda+2} .
$$

It can be seen that for $l, \lambda \rightarrow 0$ we regain the symmetric case since $\varepsilon, \chi \rightarrow 0$. The term in brackets in Eq. (51) can be interpreted as a temporal modulation of the driving term $p$ of the $z$-mode, as discussed in the main text (where the contribution $\varepsilon z$ was omitted to simplify the discussion).

\section{Conflicts of interest}

There are no conflicts to declare.

\section{Acknowledgements}

We thank Vincent Le Houerou and Fabien Montel for fruitful discussions. This article was created entirely with Free and Open-Source Software (FOSS): ImageJ/FiJi, Jupyter-Lab, QtiPlot (0.9.8), Maxima-CAS/wxMaxima, Python/Matplotlib, GNUOctave, LibreOffice Draw, Inkscape, OpenShot, Kdenlive, Lyx and TexStudio. The authors acknowledge the effort of numerous FOSS programmers without whom our work would be very difficult and much less enjoyable.

\section{Notes and references}

1 J. Elgeti, R.G. Winkler and G. Gompper, Rep. Prog. Phys., 2015, 78, 056601.

2 C. Bechinger, R. Di Leonardo, H. Löwen, C. Reichhardt, G. Volpe and G. Volpe, Rev. Mod. Phys., 2016, 88, 045006.

3 A. Bricard, J.-B. Caussin, N. Desreumaux, O. Dauchot and D. Bartolo, Nature, 2013, 503, 95-98.

4 A. Kaiser, A. Snezhko and I. S. Aranson, Sci. Adv., 2017, 3, e1601469. 
5 A. Kudrolli, G. Lumay, D. Volfson and L. S. Tsimring, Phys. Rev. Lett., 2008, 100, 058001.

6 Z. Izri, M. N. van der Linden, S. Michelin and O. Dauchot, Phys. Rev. Lett., 2014, 113, 248302.

7 A. Bouillant, T. Mouterde, P. Bourrianne, A. Lagarde, C. Clanet and D. Quéré, Nat. Phys., 2018, 14, 1188.

8 A. Baumann, A. Sánchez-Ferrer, L. Jacomine, P. Martinoty, V. Le Houerou, F. Ziebert and I. M. Kulić, Nat. Mater., 2018, 17, 523.

9 C. Ahn, K. Li and S. Cai, ACS Appl. Mater. Interfaces, 2018, 10, 25689.

10 D. Hore, A. Majumder, S. Mondal, A. Roy and A. Ghatak, Soft Matt., 2012, 8, 5038.

11 S. Mondal and A. Ghatak, Extreme Mech. Lett., 2015, 3, 24.

12 E. Efrati, E. Sharon and R. Kupferman, Soft Matt., 2013, 9, 8187.

13 T. Mura. Micromechanics of defects in solids, Vol. 3, Springer, 1987.

14 K.P. Velikov and O.D. Velev, Stabilization of Thin Films, Foams, Emulsions and Bifluid Gels with Surface-Active Solid Particles; in Colloid stability, T. F. Tadros, Ed., Wiley, 2010.
15 F. Ziebert and I.M. Kulić, Frustriert in Bewegung (Frustrated in Motion), in Physik Journal (Wiley-VCH, Weinheim), 2018, 17, 11.

16 F. Peruani, A. Deutsch and M. Bär, Phys. Rev. E, 2006, 74, 030904(R).

17 H. Linke, B.J. Alemán, L.D. Melling, M.J. Taormina, M.J. Francis, C.C. Dow-Hygelund, V. Narayanan, R.P. Taylor, and A. Stout, Phys. Rev. Lett., 2006, 96, 154502.

18 P. Illien, C. de Blois, Y. Liu, M. N. van der Linden and O. Dauchot, Phys. Rev. E, 2020, 101, 040602(R).

19 R.A. Desai, S.B. Gopal, S. Chen, C.S. Chen, J. R. Soc. Interface, 2013, 10, 20130717.

20 H. Mohrbach, A. Johner and I.M. Kulić, Phys. Rev. Lett., 2010, 105, 268102.

21 F. Ziebert, H. Mohrbach and I.M. Kulić, Phys. Rev. Lett., 2015, 114, 147101.

22 L. Bouzar, M.M. Müller, R. Messina, B. Nöding, S. Köster, H. Mohrbach, and I.M. Kulić, Phys. Rev. Lett., 2019, 122, 098101.

23 I.M. Kulić, R. Thaokar and H. Schiessel, Europhys. Lett., 2005, $72,527$. 